\title{
STEREO/SECCHI Observations on 8 December 2007: Evidence Against the Wave Hypothesis of the EIT Wave Origin
}

\author{
A.N. Zhukov $\cdot$ L. Rodriguez $\cdot$ J. de Patoul
}

Received: 7 December 2008 / Accepted: 26 April 2009 / Published online: 28 May 2009

(C) Springer Science+Business Media B.V. 2009

\begin{abstract}
The physical nature of EIT waves, large-scale bright fronts propagating in the solar corona, remains a subject of a continuing debate. Two main ways of interpreting this phenomenon have been suggested. One of them describes an EIT wave as a fast mode magnetosonic wave freely propagating in the corona. The other interpretation does not consider an EIT wave a true magnetohydrodynamic wave but instead invokes several possibilities linked to the magnetic field restructuring during the coronal mass ejection (CME) evolution in the low corona. We investigate an EIT wave observed by the SECCHI/EUVI telescopes onboard the STEREO spacecraft on 8 December 2007 . The wave front had a nearly symmetric shape and exhibited a peculiar velocity profile measured by two independent methods. After an initial short propagation at a speed of around $100 \mathrm{~km} \mathrm{~s}^{-1}$, the wave was moving at a very low velocity (around $20-40 \mathrm{~km} \mathrm{~s}^{-1}$ ) for about 30 minutes, and then was reaccelerated up to speeds of around $200 \mathrm{~km} \mathrm{~s}^{-1}$. It is difficult to envisage such a velocity change for a freely propagating coronal wave. However, such a behavior is possible, for example, for erupting prominences. We conclude that this event provides observational evidence that
\end{abstract}

STEREO Science Results at Solar Minimum

Guest Editors: Eric R. Christian, Michael L. Kaiser, Therese A. Kucera, O.C. St. Cyr.

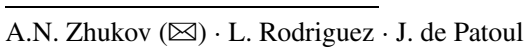

Solar-Terrestrial Center of Excellence-SIDC, Royal Observatory of Belgium, Avenue Circulaire 3,

1180 Brussels, Belgium

e-mail: Andrei.Zhukov@sidc.be

L. Rodriguez

e-mail: Luciano.Rodriguez@ sidc.be

J. de Patoul

e-mail: depatoul@mps.mpg.de

A.N. Zhukov

Skobeltsyn Institute of Nuclear Physics, Moscow State University, 119992 Moscow, Russia

J. de Patoul

Max-Planck-Institut für Sonnensystemforschung, Max-Planck-Str. 2, 37191 Katlenburg-Lindau,

Germany 
even EIT waves with a symmetric front can be produced by a magnetic field restructuring during the CME eruption.

Keywords Coronal mass ejections, low coronal signatures · Coronal mass ejections, initiation and propagation

\section{Introduction}

A long-standing problem in the physics of the solar corona is the initiation of coronal mass ejections (CMEs). The early stages of the CME initiation and development usually occur behind the coronagraph's occulter. One thus needs solar disk imaging to investigate the large-scale evolution of the low corona during the CME initiation. The value of imaging the low corona in the extreme ultraviolet (EUV) for detecting on-disk counterparts of CMEs has been well recognized since the beginning of the regular "CME Watch" observing program by the Extreme-ultraviolet Imaging Telescope (EIT; see Delaboudinière et al., 1995) onboard the Solar and Heliospheric Observatory (SOHO). The uninterrupted high-cadence observations (around 12 minutes) of the solar corona in the $195 \AA$ bandpass (showing the emission of plasma at temperatures around $1.5 \mathrm{MK}$ ) has allowed us to investigate the CME initiation in detail (e.g., Delannée, Delaboudinière, and Lamy, 2000; Hudson and Cliver, 2001).

Among the low coronal phenomena observed in association with CMEs, EIT waves (so named after EIT, the instrument used for their discovery) remain a subject of a continuing debate, especially regarding their physical nature. An EIT wave is a propagating bright front typically observed in the $195 \AA$ channel of EIT (Thompson et al., 1998). We do not consider here nonpropagating fronts that are sometimes also called EIT waves (Delannée and Aulanier, 1999; Delannée, 2000). Observations by EIT and other imaging instruments (Wills-Davey and Thompson, 1999; Zhukov and Auchère, 2004; Veronig, Temmer, and Vršnak, 2008; Long et al., 2008) demonstrated that EIT waves can also be seen in other coronal EUV bandpasses. Statistical studies showed a strong association of EIT waves with CMEs (Biesecker et al., 2002; Chen, 2006). The association of EIT waves with similar phenomena observed in H $\alpha$ (Moreton waves; see Moreton and Ramsey, 1960; Eto et al., 2002; Warmuth et al., 2004) or soft X rays (e.g., Khan and Hudson, 2000; Khan and Aurass, 2002; Narukage et al., 2002; Warmuth, Mann, and Aurass, 2005) is still unclear.

Physical mechanisms invoked to interpret EIT waves can be divided into two large groups. One of them treats EIT waves as true magnetohydrodynamic (MHD) waves. The propagation of EIT waves along the solar surface (i.e., across the approximately vertical coronal magnetic field) led to the hypothesis that an EIT wave is a fast-mode magnetosonic wave. This interpretation is insensitive to the exact process of the wave generation. It may be a pressure pulse associated with a solar flare (Wu et al., 2001; see also the Moreton wave model by Uchida, 1968), or any short-lived disturbance produced by a CME (Wang, 2000), for example, the initial lateral CME expansion that lasts for a limited time. Any such perturbation would propagate across the magnetic field lines as a fast magnetosonic wave. It is essential that this wave be a freely propagating wave. It may or may not steepen to form a fast mode shock (Vršnak and Lulić, 2000; Pomoell, Vainio, and Kissmann, 2008). The fast magneotosonic wave hypothesis (especially that of a wave produced by a flare) is a natural explanation of the often-circular shape of EIT wave fronts. Arguments against the wave models point at unrealistically low values of the Alfvén speed in the quiet Sun regions (Wills-Davey, DeForest, and Stenflo, 2007) and often complicated morphologies of the EIT wave front that look incompatible with a simple fast MHD wave (Delannée, 2000; Zhukov and Auchère, 2004). 
Another point of view suggests that an EIT wave is not a true MHD wave but is instead produced by the restructuring of the coronal magnetic field during initial stages of the CME eruption. In the model by Chen et al. (2002), an EIT wave is a compression region produced by the successive stretching (a term we prefer to use instead of "opening") of expanding magnetic field lines during the CME lifting. Delannée et al. (2008) have developed a model in which an EIT wave is due to the compression and heating in expanding electric current sheets at the boundary between the erupting flux rope and the ambient magnetic field. In both cases the position of the EIT wave front is closely linked to the rate of the CME's lateral expansion. This point of view, however, has a difficulty in explaining an almost circular (symmetric) shape of many EIT waves (including a classical example of 12 May 1997 described by Thompson et al., 1998). The model by Chen et al. (2002) is two dimensional and needs to be extended to three dimensions to explain the shape of the wave in the realistic three-dimensional geometry. In the model by Delannée et al. (2008) the initial dimensions of the erupting flux region were chosen in an ad hoc way to make the outer boundary of the expanding flux rope nearly circular (when seen from the top). The fast MHD wave hypothesis still looks very attractive for description of EIT waves with symmetric fronts. It is also possible that EIT waves in different events may be produced by different mechanisms (Zhukov and Auchère, 2004).

In this paper we present the observations of an EIT wave event on 8 December 2007 that was detected by SOHO/EIT and the Extreme Ultraviolet Imager (EUVI), which is a part of the Sun Earth Connection Coronal and Heliospheric Investigation (SECCHI) suite (see Howard et al., 2008) onboard the Solar TErrestrial RElations Observatory (STEREO) mission (see Kaiser et al., 2008). In Section 2 we summarize the observations of the EIT wave that had a symmetric front (i.e., it was propagating in all directions). We demonstrate that its peculiar velocity profile can hardly be explained by the fast magnetosonic wave hypothesis. We discuss our results and draw conclusions in Section 3.

\section{Observations}

We investigate the eruption that occurred on 8 December 2007, approximately between 17:00 UT and 19:00 UT. Both SOHO and STEREO missions observed this event in detail. The eruption source region (NOAA H $\alpha$ plage 10977 without sunspots) was located at S05W26, as seen from the Earth (and SOHO), at Carrington longitude $114^{\circ}$. The STEREO Ahead (A) and Behind (B) spacecraft were situated $20.8^{\circ}$ to the west and $21.7^{\circ}$ to the east of the Earth, respectively. Therefore, the $\mathrm{H} \alpha$ plage 10977 was located close to the central meridian (approximately at W05) as seen from STEREO A and far to the west (approximately at W48) as seen from STEREO B. As seen in the EUV, the corona above the H $\alpha$ plage had a typical appearance of a small active region (see Figure 1a), so we will refer to it as to an active region throughout the rest of the paper.

The eruption was not associated with a reported X-ray flare, although there was an increase of the solar X-ray flux up to the A8.3 level (with the background at A4.2), peaking at 18:13 UT (as detected by GOES-11). A typical postflare arcade was detected in the SEC$\mathrm{CHI} / \mathrm{EUVI}$ data starting around 18:15 UT (Figure 1b). The visible-light coronagraph COR1 of the SECCHI suite (Howard et al., 2008) has detected an associated CME that first appeared in the COR1 B field of view at 18:45 UT. It is interesting to note that the same CME looks very weak in COR1 images taken from the A spacecraft. This corresponds well to the apparent difference in the CME source region position as seen from the two STEREO spacecraft: close to the disk center as seen from STEREO A and around $40^{\circ}$ from the limb as seen from STEREO B. Thomson scattering is more efficient for structures located closer 

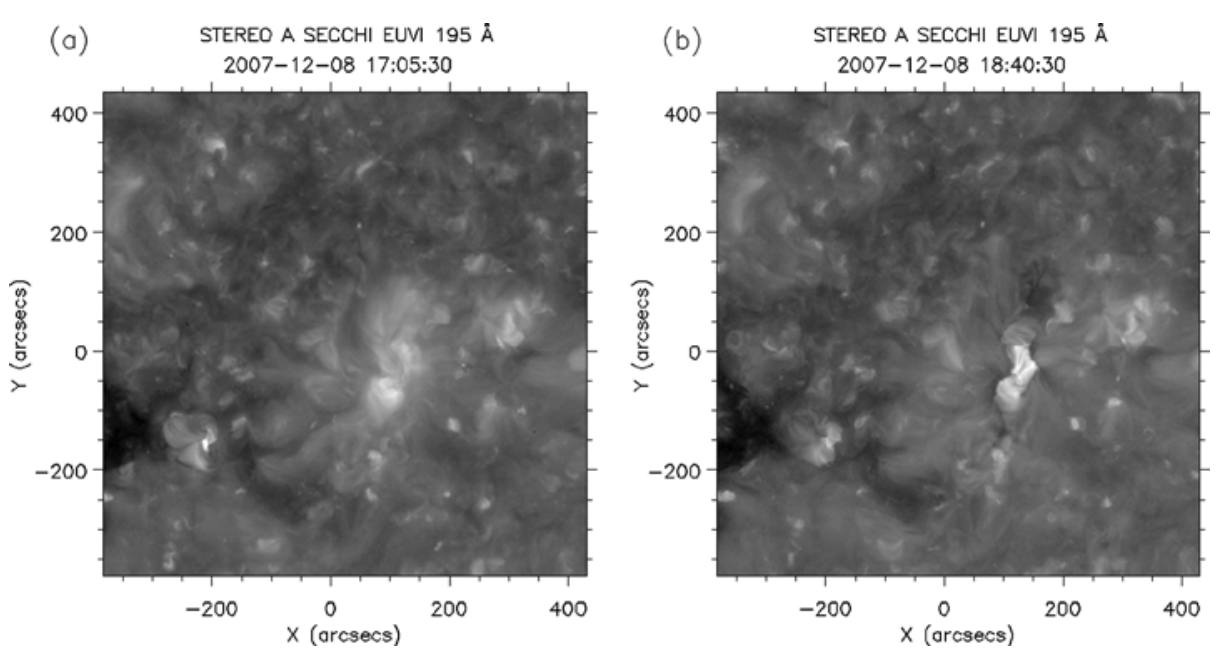

Figure 1 NOAA H $\alpha$ plage 10977 observed by STEREO A SECCHI/EUVI in the $195 \AA$ bandpass on 8 December 2007. Panels (a) and (b) show the coronal morphology before and after the eruption.

to the plane of the sky (Billings, 1966). According to the Large Angle Spectroscopic Coronagraph (LASCO; see Brueckner et al., 1995) CME catalog ${ }^{1}$ (Yashiro et al., 2004), the CME speed was around $348 \mathrm{~km} \mathrm{~s}^{-1}$ (projected on the SOHO plane of the sky), the CME central position angle was $265^{\circ}$, and the CME angular width was $79^{\circ}$.

The SECCHI/EUVI data were taken at a higher cadence than the SOHO/EIT data: 10 minutes versus 12 minutes in the $195 \AA$ bandpass and 2.5 minutes versus 6 hours in the $171 \AA$ bandpass. EUVI data thus give us an opportunity to observe the EIT wave nearly simultaneously in two bandpasses (171 and $195 \AA$ ) at a cadence higher than that of EIT, so we will use the EUVI data in this study. The dynamics of the wave front propagation can then be studied in detail (see also Veronig, Temmer, and Vršnak, 2008). The cadence of observations in the $284 \AA$ EUVI bandpass was lower (20 minutes) and the wave in the $304 \AA$ bandpass was not visible, so the data taken in these two bandpasses are not considered here. Owing to the position of the active region close to the disk center as seen from STEREO A (which minimizes the projection effects) and to the higher spatial resolution of the EUVI telescope onboard STEREO A (compared to STEREO B) because of its closer distance to the Sun (0.966662 AU for the A spacecraft versus 1.025682 AU for the B spacecraft), we describe here the propagation of the EIT wave as seen by SECCHI/EUVI A. We, however, checked the EUVI B data (as well as SOHO/EIT data) and found that the behavior of the EIT wave was similar in all three data sets.

The EIT wave was well visible both in the 195 and $171 \AA$ A bandpasses of EUVI (Figure 2). The temperature response of both bandpasses is relatively broad and overlapping (Howard et al., 2008), with the $171 \AA$ (195 $\AA$ ) bandpass sensitivity peaking at temperatures around $1 \mathrm{MK}(1.5 \mathrm{MK})$. The cadence in the $171 \AA$ channel was significantly better than that in the $195 \AA$ channel (2.5 and 10 minutes, respectively), but the wave front contrast was higher in the $195 \AA$ bandpass. This is in agreement with the result obtained by Wills-Davey and Thompson (1999). Figure 3 shows the propagation of the EIT wave in running-difference images (in which the previous image was subtracted from every image) as observed in the

\footnotetext{
${ }^{1}$ Available online at http://cdaw.gsfc.nasa.gov/CME_list/.
} 

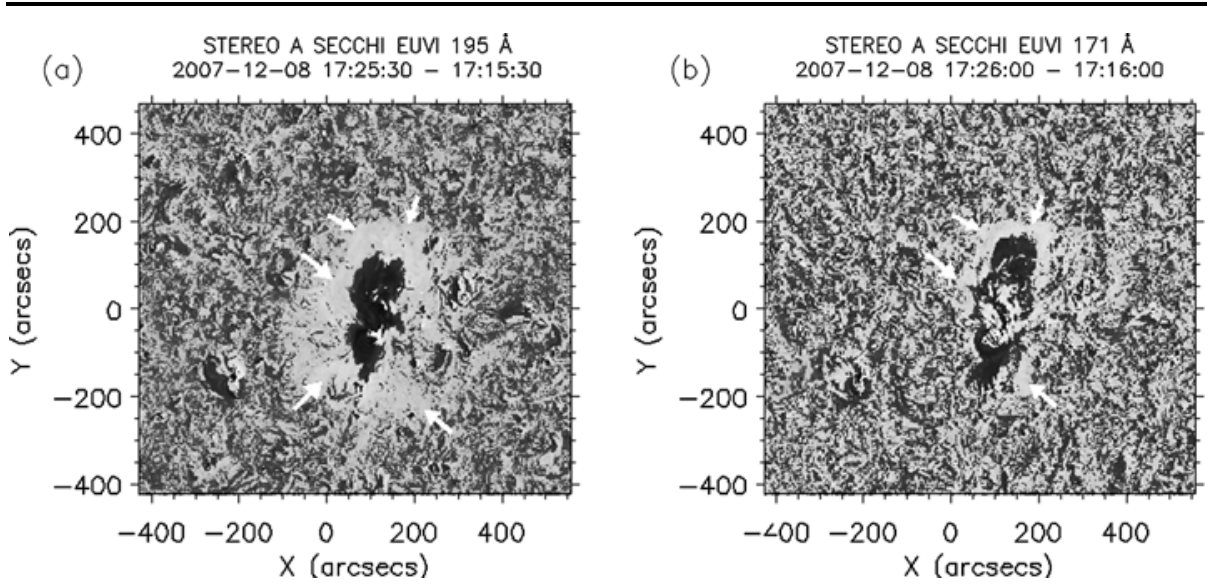

Figure 2 STEREO A SECCHI/EUVI difference images of the EIT wave event on 8 December 2007. Panels (a) and (b) show the wave front seen in the 195 and $171 \AA$ bandpasses, respectively. The image contrast was increased to make the weak wave front (indicated by white arrows) more visible.

$195 \AA$ bandpass. The wave is seen propagating in all directions from the active region and is thus typical for events with a rather symmetric wave front (although the shape of the front is not circular but rather ellipsoidal at early stages of its propagation). Nearly simultaneous images taken in the 171 and $195 \AA$ bandpasses (Figure 2) demonstrate a certain correspondence of wave front positions observed in both bandpasses, especially to the north of the active region, although some parts of the wave front to the east and south of the active region are almost invisible in the $171 \AA$ bandpass.

We chose a direction (toward the north-northeast from the active region; see Figure 4) along which the wave front is well visible in both bandpasses up to the largest distances from the initiation site. This direction is representative enough as the wave front trajectories along other directions look similar. We identified the wave front positions in every image. The wave front is wide (around $40 \mathrm{Mm}$ as seen in the $171 \AA$ a bandpass and around $80 \mathrm{Mm}$ as seen in the $195 \AA$ bandpass), so we measured the position of the peak intensity across each front and tracked its propagation in the chosen direction along the great circle on the solar surface.

To increase the signal-to-noise ratio, we have rebinned the original images (of $2048 \times$ 2048 pixels) to the size of $1024 \times 1024$ pixels. The errors of our measurements are accounted for by intrinsic uncertainty from the finite width of the weak, diffuse wave front, which is often difficult to measure because of the noise. We estimate conservatively the error bars of our measurements to be \pm 2 rebinned pixels (i.e., four EUVI pixels). One EUVI A pixel corresponds to 1.6 arc sec (Howard et al., 2008), which on the day of observations amounts to $1100 \mathrm{~km}$ at the solar disk center. We did not correct for solar rotation since it is difficult to do given the finite (unknown) height range of the front. We estimate the solar rotation to introduce uncertainties of the order of $300 \mathrm{~km}$ in 2.5 minutes (the cadence of observations in the $171 \AA$ bandpass), which is smaller than our error bars.

Figure 5 shows the distance-time plot for the wave propagation, in both the 171 and $195 \AA$ bandpasses. The distances are measured from the point marked by a symbol " 0 " in Figure 4 (but this point is not taken into account for speed calculations in the following). The positions of the wave front in two bandpasses are very close in nearly simultaneous images (separated by 30 seconds). The only exception may be our last measurement. The wave front in the $171 \AA$ bandpass can be last detected at 18:18:30 UT, whereas it is still 

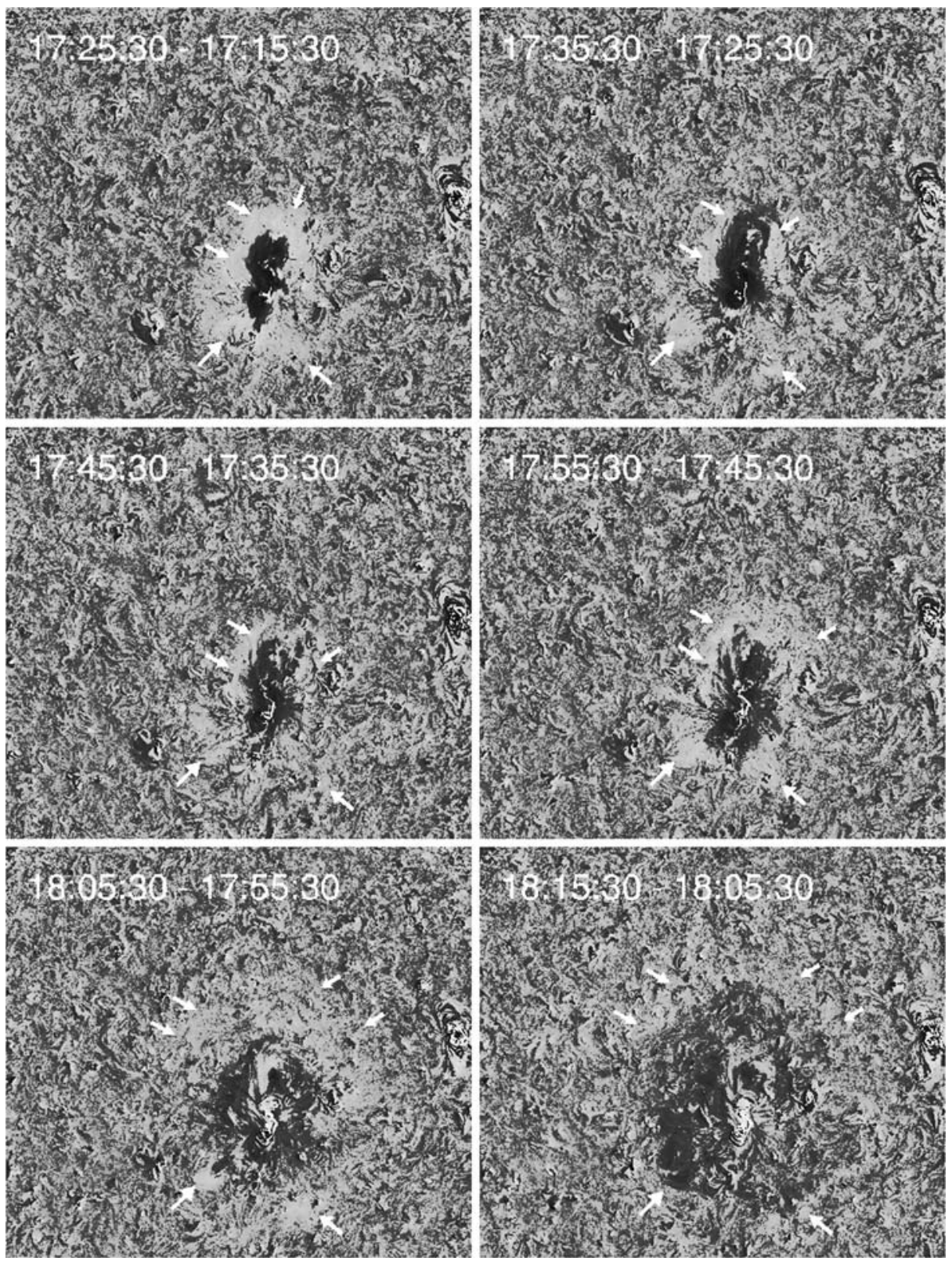

Figure 3 STEREO A SECCHI/EUVI 195 Å running difference images of the EIT wave event on 8 December 2007. The image contrast was increased to make the weak wave front (indicated by white arrows) more visible.

well visible in the $195 \AA$ a bandpass at 18:20:30 UT. Around that time the front became very weak in the $171 \AA$ bandpass, so its position is rather uncertain. These last measurements of the wave front position around 18:18-18:20 UT are not significant for the overall wave propagation. 
Figure 4 A part of the STEREO A SECCHI/EUVI $195 \AA$ difference image showing the position of the EIT wave front on 8 December 2007 at $17: 25: 30$ UT. The dark arc is the solar limb. The symbol " 0 " marks the point from which the distances are measured and the cross marks the position of the EIT wave front along the great circle on the solar surface shown with a white line.
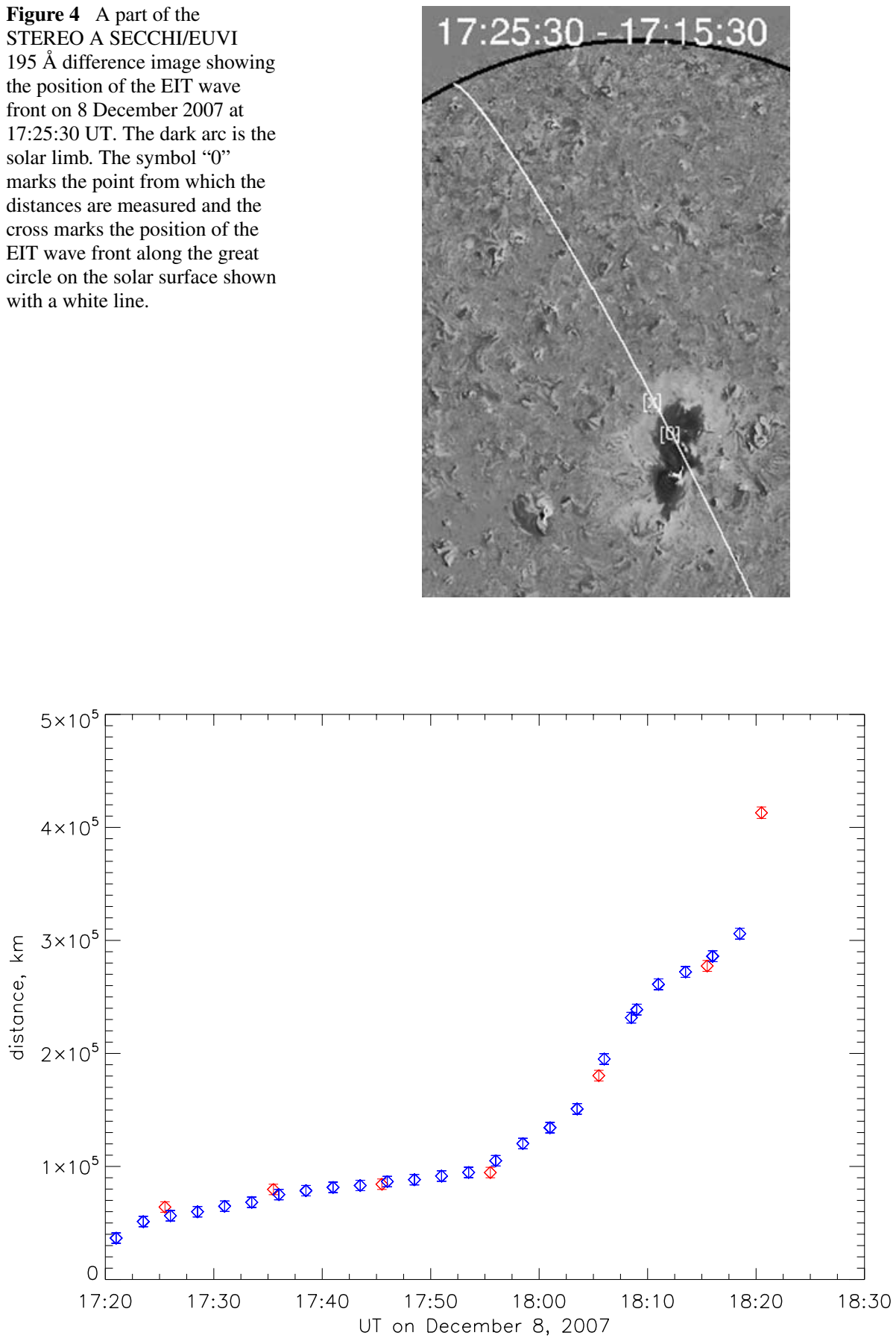

Figure 5 Distance-time plot of the EIT wave propagation on 8 December 2007. The measurements were performed in the north-northeast direction along the white line shown in Figure 4. Red (blue) symbols denote the EIT wave front position observed in the $195 \AA$ (171 $\AA$ ) bandpass of the SECCHI/EUVI telescope onboard STEREO A. 


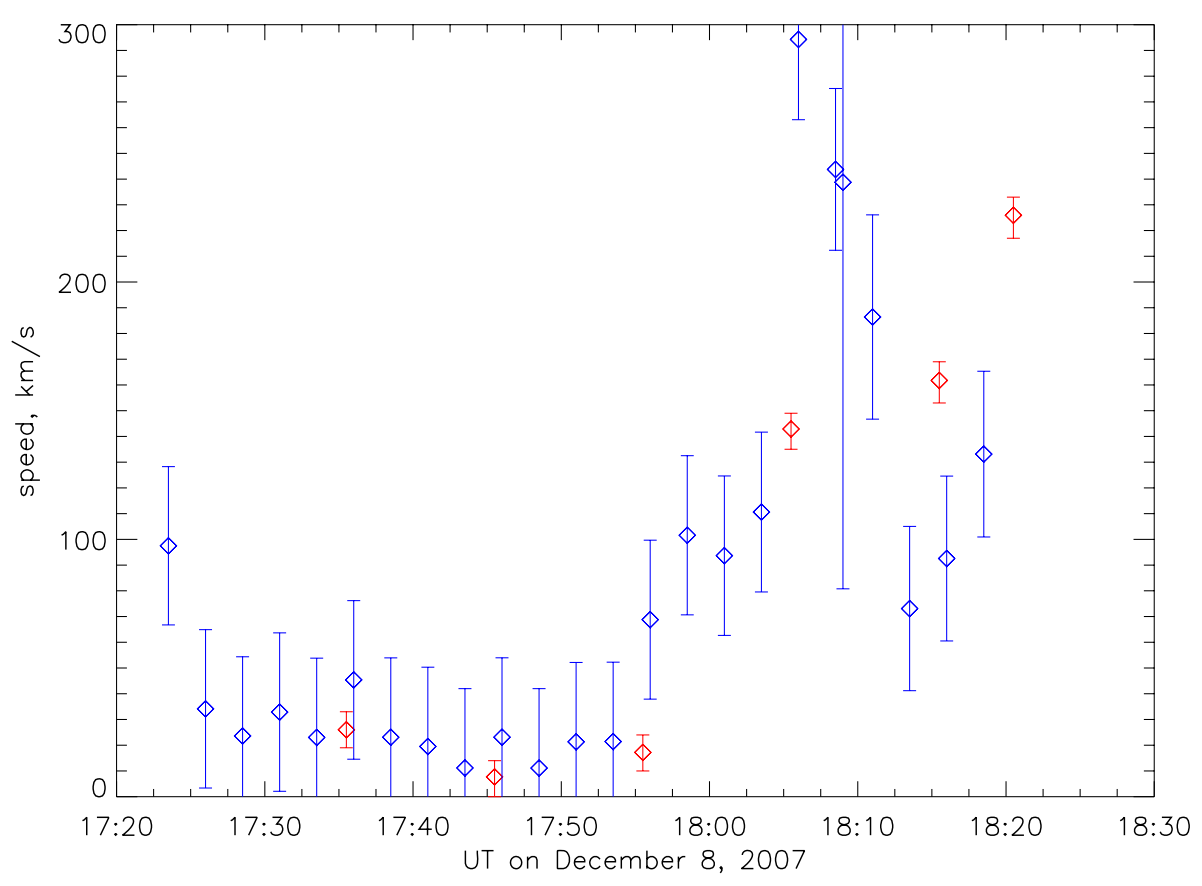

Figure 6 Velocity - time plot of the EIT wave propagation on 8 December 2007. EIT wave speeds calculated from consecutive measurements of the wave front position (shown in Figure 5) separately in the $195 \AA$ (171 bandpass of the SECCHI/EUVI telescope onboard STEREO A are represented by red (blue) symbols.

Figure 6 shows the velocity - time plot. The wave speeds were derived from consecutive wave positions in both bandpasses separately. A higher uncertainty for the speed at 18:09:00 UT results from measurements of the wave front position in two $171 \AA$ images taken with the interval of 30 seconds only (18:08:30 and 18:09:00 UT). It is clear from Figures 5 and 6 that the wave does not propagate at a constant velocity. Initially the wave is observed propagating at a speed of around $100 \mathrm{~km} \mathrm{~s}^{-1}$. Shortly afterward (in around 3 minutes), the wave decelerated significantly. For almost 30 minutes, the wave front speed was around $20-30 \mathrm{~km} \mathrm{~s}^{-1}$, which is close to a speed of zero if one takes into account the error bars (see Figure 6). Around 17:55 UT the wave was reaccelerated and reached velocities around $300 \mathrm{~km} \mathrm{~s}^{-1}$. This speed is rather typical for EIT waves (see, e.g., Klassen et al., 2000). After 18:10 UT the wave speed dropped to values below $200 \mathrm{~km} \mathrm{~s}^{-1}$.

The high-cadence data taken in the $171 \AA$ bandpass allow us to use the technique of Huygens plotting (Wills-Davey and Thompson, 1999; Wills-Davey, 2006) to measure the evolution of the EIT wave speed in detail. We corrected the $171 \AA$ images for the photospheric differential rotation by de-rotating all images to 17:55:30 UT (approximately the middle of the event). We then manually traced the wave front in every image (white lines in Figure 7). We concentrated on the area to the north of the active region because the EIT wave front is the strongest there and is visible up to the largest distances from the initiation site.

We selected several representative points on the first front and tracked their trajectories during the EIT wave propagation. By assuming that the wave front travels as efficiently as possible, every selected point of the first front is mapped to the closest point in the next 
Figure 7 Huygens plotting of the EIT wave front propagation for the event of

8 December 2007. White lines indicate the positions of the $171 \AA$ fronts over time (with the propagation direction being outward from the active region). The four labeled black lines follow trajectories of four wave front points. The background is a part of the STEREO A SECCHI/EUVI $171 \AA$ image taken at 17:23:30 UT and rotated to $17: 55: 30 \mathrm{UT}$.

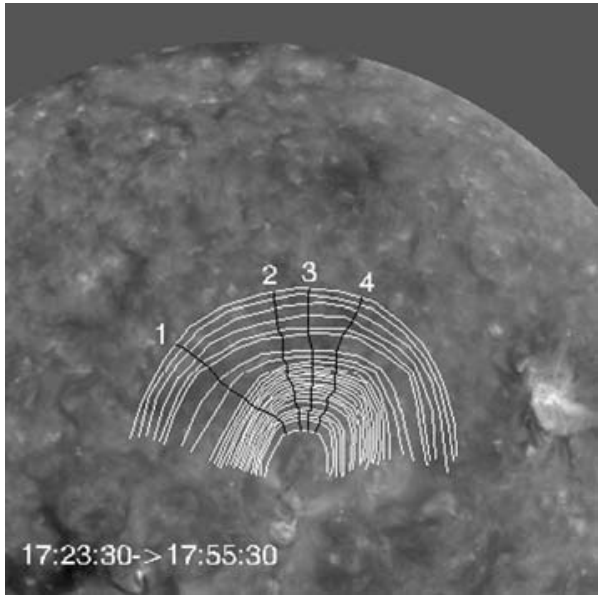

front. This procedure is continued until the last front is reached. We avoided selecting points close to the ends of the tracked fronts. The resulting trajectories are shown with black lines in Figure 7. The speeds were calculated along the trajectories of these four points and the speed evolution is plotted in Figure 8. The speed error bars were calculated as previously described.

Comparing four panels of Figure 8, we can readily see that the velocity evolution for different points is qualitatively the same. The Huygens plotting technique independently confirms measurements shown in Figure 6 as the velocity - time profiles in both figures are very similar. The initial speed is around $100 \mathrm{~km} \mathrm{~s}^{-1}$. Shortly after the start, the wave speed decreases to around $30-40 \mathrm{~km} \mathrm{~s}^{-1}$ and stays at this level for around 30 minutes. Then the wave is accelerated to speeds of about $200 \mathrm{~km} \mathrm{~s}^{-1}$, and after reaching this peak value the wave speed decreases again. The final observed values of the speed are around $100 \mathrm{~km} \mathrm{~s}^{-1}$. The speed evolution for other points that may be selected on the first front is very similar.

We note that the wave speed is difficult to measure for the last two or three fronts as the wave becomes very weak. Another uncertainty is the speed value at 18:09:00 UT. The wave speed at that moment is off the scale of Figure 8 for all four selected points. This is, however, most probably an artefact since the previous image was exceptionally taken only 30 seconds earlier (at 18:08:30 UT). The displacement of the diffuse front during so short a time is difficult to measure against the noisy background and the speed is thus subject to significant measurement errors. We also note that whereas the temporal resolution of observations in the $171 \AA$ bandpass ( 2.5 minutes) is increased by almost a factor of five in comparison with a typical EIT cadence (12 minutes), the spatial resolution is nearly the same since we had to rebin EUVI images to $1024 \times 1024$ pixels to increase a weak signalto-noise ratio. Insufficient spatial resolution limits the precision of the Huygens plotting technique, which was successfully applied to high-cadence, high-spatial-resolution TRACE observations (Wills-Davey and Thompson, 1999; Wills-Davey, 2006).

The velocity profiles shown in Figures 6 and 8 are unusual for EIT waves. Constant deceleration (Warmuth et al., 2004; Veronig, Temmer, and Vršnak, 2008), nearly constant speed (White and Thompson, 2005), or irregular velocity profiles (Wills-Davey and Thompson, 1999) were reported for waves observed in $\mathrm{H} \alpha$, EUV, and radio emission. But the velocity change we describe here - decelerating nearly to zero after a short propagation and then accelerating again - is observed, to our knowledge, for the first time. 
Figure 8 Velocity-time plot of the EIT wave propagation observed in the $171 \AA$ bandpass on 8 December 2007. The speeds are calculated from consecutive measurements of the wave front position using Huygens plotting. The four panels correspond to the four trajectories labeled in Figure 7.

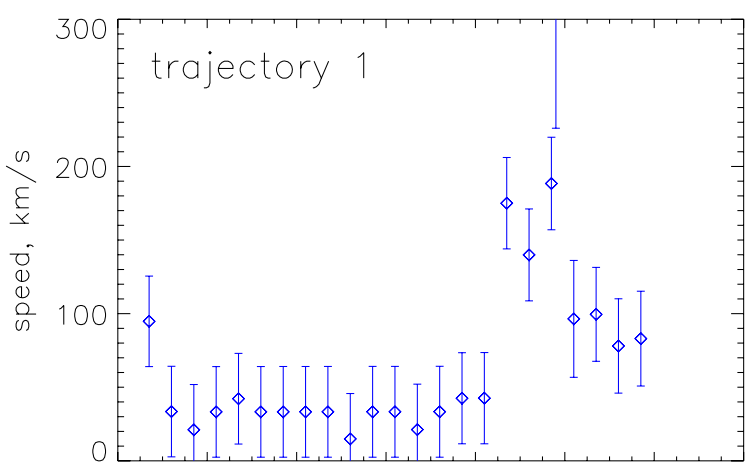

17:20 17:30 17:40 17:50 18:00 18:10 18:20 18:30

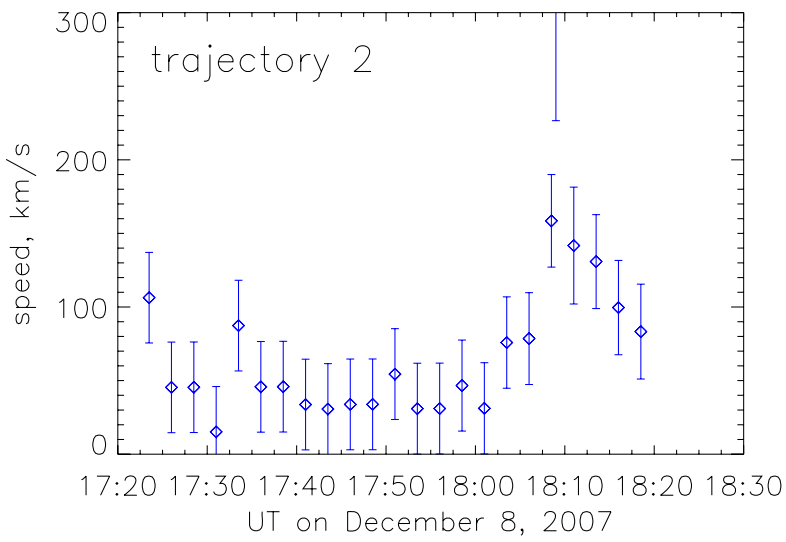

\section{Discussion and Conclusions}

We now try to interpret the wave front propagation described in Section 2 using different EIT wave models. Explaining the velocity profile of our event (Figure 6) in the framework of the fast mode MHD wave appears to be very difficult. The propagation speed of the fast magnetosonic wave across the magnetic field is always higher than the Alfvén speed. The trajectory shown in Figure 5 should then reflect a rather special distribution of magnetic field strength or coronal density around the active region, so that the local Alfvén speed drops strongly to very low values (around $20 \mathrm{~km} \mathrm{~s}^{-1}$ ). Such values of the Alfvén speed are not reported for the low coronal heights where EIT waves usually propagate (see, e.g., Evans et al., 2008). Indeed, taking order of magnitude values for the coronal magnetic field in the quiet Sun, $B=1 \mathrm{G}$, and a rather high (for the quiet Sun regions) density $n=10^{9} \mathrm{~cm}^{-3}$, we can estimate an Alfvén speed of $v_{\mathrm{A}}=B / \sqrt{4 \pi m_{\mathrm{p}} n} \sim 70 \mathrm{~km} \mathrm{~s}^{-1}$ (where $m_{\mathrm{p}}$ is the proton mass). To reduce the Alfvén speed below that value would require either a further (unrealistic) increase of the coronal density or a decrease of coronal magnetic field. Figure 9 shows the Michelson and Doppler Imager (MDI; see Scherrer et al., 1995) pre-event photospheric magnetogram of the quiet Sun around the active region (where the observed EIT wave nearly stopped) and a co-spatial EIT image of the corona taken nearly simultaneously in the $195 \AA$ bandpass. It demonstrates that the quiet Sun around the active region has usual morphologies both in the photospheric magnetic field distribution and in the distribution of dense coronal structures. No peculiar structures in the density (e.g., very bright regions) or 
Figure 8 (Continued.)

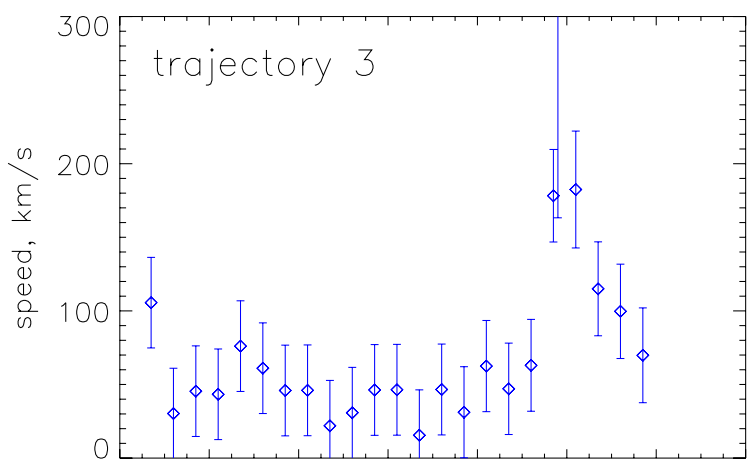

17:20 17:30 17:40 17:50 18:00 18:10 18:20 18:30

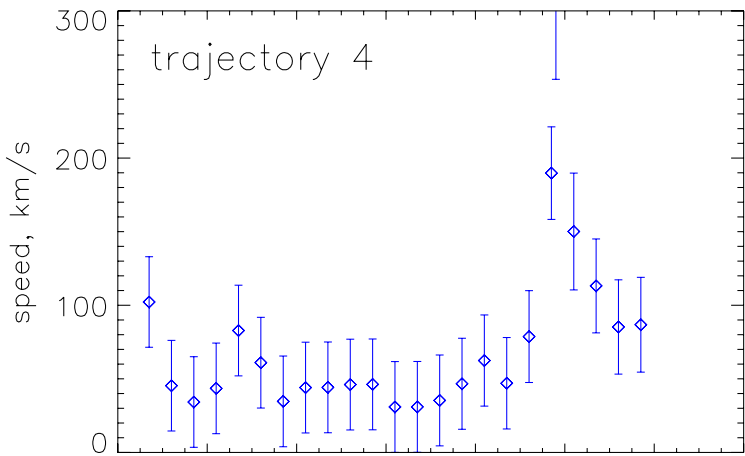

17:20 17:30 17:40 17:50 18:00 18:10 18:20 18:30 UT on December 8, 2007
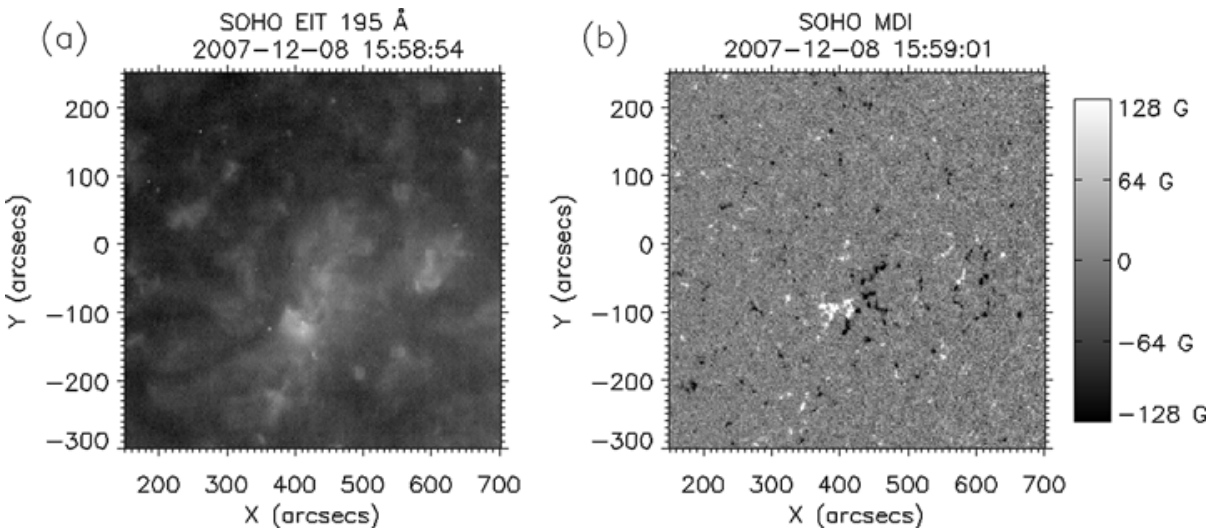

Figure 9 Nearly simultaneous images of the NOAA H $\alpha$ plage 10977 taken by SOHO/EIT in the $195 \AA$ A bandpass (a) and by SOHO/MDI (b). The bar on the right shows the scaling of the magnetic field strength in the MDI image. 
magnetic field (very weak fields) can be noticed. We thus conclude that the EIT wave in this event cannot be a fast-mode freely propagating MHD wave.

On the contrary, such a peculiar speed behavior can be explained if we assume that the EIT wave in our event is not a true wave but is instead linked to the CME eruption. An EIT wave in this interpretation may represent the compressed or heated material at the outer edge of the CME flanks (Chen et al., 2002; Delannée et al., 2008). A plausible scenario of the event can then be outlined as follows. The main magnetic flux system of the CME starts erupting and in a short time reaches a certain height in the low corona. The initial stage of the eruption can be accompanied by the lateral expansion as well (e.g., Delannée et al., 2008). The EIT wave at the outer boundary of the CME will also then reach a certain distance from the active region. Then the CME ascent may slow and nearly stop. Such a behavior is not unusual. Romano, Contarino, and Zuccarello (2003) have reported observations of an erupting prominence that was initially destabilized by the kink instability and rose to a certain height above the solar surface. It briefly stopped there but did not find a new equilibrium configuration and subsequently erupted. The height - time curve in Figure 5 by Romano, Contarino, and Zuccarello (2003) is remarkably similar to our Figure 5: the prominence rises during the activation phase, remains at more or less the same height during the following 15 minutes, and then continues its upward motion (eruption phase). In such a situation the speed profile of the horizontal (along the solar surface) propagation of the EIT wave may resemble the speed profile of the vertical (ascending) motion of the erupting filament. We note that this scenario works regardless of the precise relation of the EIT wave to the CME; see the models of Chen et al. (2002) and Delannée et al. (2008).

The EIT wave front in our event propagates in all directions (i.e., the wave front is rather symmetric). Our results provide a strong indication that even a symmetric wave front shape can be present in EIT waves produced by CMEs. The electric current shell model by Delannée et al. (2008) can describe such symmetric wave front shapes. A fully three dimensional modeling of the EIT wave produced via the mechanism suggested by Chen et al. (2002) is still not available.

In conclusion, our analysis of the symmetric EIT wave event on 8 December 2007 observed at high spatial and temporal resolution by the SECCHI/EUVI telescope onboard STEREO allows us to discard the freely propagating fast-mode MHD wave as a possible source of the EIT wave disturbance in this event. Our results suggest that the explanation of the EIT wave physical nature in terms of compression and/or heating at the expanding CME flanks is more plausible.

Acknowledgements The STEREO/SECCHI data used here were produced by an international consortium of the Naval Research Laboratory (USA), Lockheed Martin Solar and Astrophysics Lab (USA), NASA Goddard Space Flight Center (USA), Rutherford Appleton Laboratory (UK), University of Birmingham (UK), Max-Planck-Institut for Solar System Research (Germany), Centre Spatiale de Liège (Belgium), Institut d'Optique Théorique et Appliquée (France), and Institut d'Astrophysique Spatiale (France). EIT and MDI data have been used courtesy of the SOHO/EIT and SOHO/MDI consortiums, respectively. SOHO is a project of international cooperation between ESA and NASA. The authors acknowledge support from the Belgian Federal Science Policy Office through the ESA - PRODEX programme.

\section{References}

Biesecker, D.A., Myers, D.C., Thompson, B.J., Hammer, D.M., Vourlidas, A.: 2002, Astrophys. J. 569, 1009. Billings, D.E.: 1966, A Guide to the Solar Corona, Academic Press, San Diego.

Brueckner, G.E., Howard, R.A., Koomen, M.J., Korendyke, C.M., Michels, D.J., Moses, J.D., Socker, D.G., Dere, K.P., Lamy, P.L., Llebaria, A., Bout, M.V., Schwenn, R., Simnett, G.M., Bedford, D.K., Eyles, C.J.: 1995, Solar Phys. 162, 357. 
Chen, P.F.: 2006, Astrophys. J. 641, 153.

Chen, P.F., Wu, S.T., Shibata, K., Fang, C.: 2002, Astrophys. J. 572, 99.

Delaboudinière, J.P., Artzner, G.E., Brunaud, J., Gabriel, A.H., Hochedez, J.F., Millier, F., Song, X.Y., Au, B., Dere, K.P., Howard, R.A., Kreplin, R., Michels, D.J., Moses, J.D., Defise, J.M., Jamar, C., Rochus, P., Chauvineau, J.P., Marioge, J.P., Catura, R.C., Lemen, J.R., Shing, L., Stern, R.A., Gurman, J.B., Neupert, W.M., Maucherat, A., Clette, F., Cugnon, P., van Dessel, E.L.: 1995, Solar Phys. 162, 291.

Delannée, C.: 2000, Astrophys. J. 545, 512.

Delannée, C., Aulanier, G.: 1999, Solar Phys. 190, 107.

Delannée, C., Delaboudinière, J.P., Lamy, P.: 2000, Astron. Astrophys. 355, 725.

Delannée, C., Török, T., Aulanier, G., Hochedez, J.F.: 2008, Solar Phys. 247, 123.

Eto, S., Isobe, H., Narukage, N., Asai, A., Morimoto, T., Thompson, B., Yashiro, S., Wang, T., Kitai, R., Kurokawa, H., Shibata, K.: 2002, Publ. Astron. Soc. Japan 54, 481.

Evans, R.M., Opher, M., Manchester, W.B., Gombosi, T.I.: 2008, Astrophys. J. 687, 1355.

Howard, R.A., Moses, J.D., Vourlidas, A., Newmark, J.S., Socker, D.G., Plunkett, S.P., Korendyke, C.M., Cook, J.W., Hurley, A., Davila, J.M., Thompson, W.T., St. Cyr, O.C., Mentzell, E., Mehalick, K., Lemen, J.R., Wuelser, J.P., Duncan, D.W., Tarbell, T.D., Wolfson, C.J., Moore, A., Harrison, R.A., Waltham, N.R., Lang, J., Davis, C.J., Eyles, C.J., Mapson-Menard, H., Simnett, G.M., Halain, J.P., Defise, J.M., Mazy, E., Rochus, P., Mercier, R., Ravet, M.F., Delmotte, F., Auchere, F., Delaboudiniere, J.P., Bothmer, V., Deutsch, W., Wang, D., Rich, N., Cooper, S., Stephens, V., Maahs, G., Baugh, R., McMullin, D., Carter, T.: 2008, Space Sci. Rev. 136, 67.

Hudson, H.S., Cliver, E.W.: 2001, J. Geophys. Res. 106, 25199.

Kaiser, M.L., Kucera, T.A., Davila, J.M., St. Cyr, O.C., Guhathakurta, M., Christian, E.: 2008, Space Sci. Rev. 136, 5.

Khan, J.I., Aurass, H.: 2002, Astron. Astrophys. 383, 1018.

Khan, J.I., Hudson, H.S.: 2000, Geophys. Res. Lett. 27, 1083.

Klassen, A., Aurass, H., Mann, G., Thompson, B.J.: 2000, Astron. Astrophys. Suppl. 141, 357.

Long, D.M., Gallagher, P.T., McAteer, R.T.J., Bloomfield, D.S.: 2008, Astrophys. J. 680, 81.

Moreton, G.E., Ramsey, H.E.: 1960, Publ. Astron. Soc. Pac. 72, 357.

Narukage, N., Hudson, H.S., Morimoto, T., Akiyama, S., Kitai, R., Kurokawa, H., Shibata, K.: 2002, Astrophys. J. 572, 109.

Pomoell, J., Vainio, R., Kissmann, R.: 2008, Solar Phys. 253, 249.

Romano, P., Contarino, L., Zuccarello, F.: 2003, Solar Phys. 214, 313.

Scherrer, P.H., Bogart, R.S., Bush, R.I., Hoeksema, J.T., Kosovichev, A.G., Schou, J., Rosenberg, W., Springer, L., Tarbell, T.D., Title, A., Wolfson, C.J., Zayer, I., MDI Engineering Team: 1995, Solar Phys. 162, 129.

Thompson, B.J., Plunkett, S.P., Gurman, J.B., Newmark, J.S., St. Cyr, O.C., Michels, D.J.: 1998, Geophys. Res. Lett. 25, 2465.

Uchida, Y.: 1968, Solar Phys. 4, 30.

Veronig, A.M., Temmer, M., Vršnak, B.: 2008, Astrophys. J. 681, 113.

Vršnak, B., Lulić, S.: 2000, Solar Phys. 196, 157.

Wang, Y.M.: 2000, Astrophys. J. 543, 89.

Warmuth, A., Mann, G., Aurass, H.: 2005, Astrophys. J. 626, 121.

Warmuth, A., Vršnak, B., Magdalenić, J., Hanslmeier, A., Otruba, W.: 2004, Astron. Astrophys. 418, 1101.

White, S.M., Thompson, B.J.: 2005, Astrophys. J. 620, 63.

Wills-Davey, M.J.: 2006, Astrophys. J. 645, 757.

Wills-Davey, M.J., Thompson, B.J.: 1999, Solar Phys. 190, 467.

Wills-Davey, M.J., DeForest, C.E., Stenflo, J.O.: 2007, Astrophys. J. 664, 556.

Wu, S.T., Zheng, H., Wang, S., Thompson, B.J., Plunkett, S.P., Zhao, X.P., Dryer, M.: 2001, J. Geophys. Res. 106, 25089.

Yashiro, S., Gopalswamy, N., Michalek, G., St. Cyr, O.C., Plunkett, S.P., Rich, N.B., Howard, R.A.: 2004, J. Geophys. Res. 109, A07105.

Zhukov, A.N., Auchère, F.: 2004, Astron. Astrophys. 427, 705. 